\title{
Interference Suppression Performance of Automotive UWB Radars Using Pseudo Random Sequences
}

\author{
Idnin PASYA ${ }^{1}$, Hiroshi KATO ${ }^{2}$, Takehiko KOBAYASHI ${ }^{2}$ \\ ${ }^{1}$ Microwave Technology Centre, Faculty of Electrical Engineering, Universiti Teknologi MARA, 40450 Shah Alam, \\ Selangor, Malaysia \\ ${ }^{2}$ Wireless Systems Laboratory, Tokyo Denki University, 5-Senju Asahi-Cho, Adachi-ku, Tokyo 120-8551, Japan \\ idnin@salam.uitm.edu.my, koba@c.dendai.ac.jp
}

\begin{abstract}
Ultra wideband (UWB) automotive radars have attracted attention from the viewpoint of reducing traffic accidents. The performance of automotive radars may be degraded by interference from nearby radars using the same frequency. In this study, a scenario where two cars pass each other on a road was considered. Considering the utilization of cross-polarization, the desired-to-undesired signal power ratio (DUR) was found to vary approximately from -10 to $30 \mathrm{~dB}$. Different pseudo random sequences were employed for spectrum spreading the different radar signals to mitigate the interference effects. This paper evaluates the interference suppression provided by maximum length sequence (MLS) and Gold sequence (GS) through numerical simulations of the radar's performance in terms of probability of false alarm and probability of detection. It was found that MLS and GS yielded nearly the same performance when the DUR is $-10 \mathrm{~dB}$ (worst case); for example when fixing the probability of false alarm to $10^{-4}$, the probabilities of detection were 0.964 and 0.946 respectively. The GS are more advantageous than MLS due to larger number of different sequences having the same length in GS than in MLS.
\end{abstract}

\section{Keywords}

UWB, radar, maximum length sequence, Gold sequence, interference suppression, detection probability

\section{Introduction}

Ultra wideband (UWB) automotive radars are receiving huge research interests for its potential to be applied as automotive radars that could assists drivers, as well as automated driving applications [1]. In comparison with conventional vehicular sensing system using infra-red, ultra-sound, and millimeter-waves radar, the Ka-band UWB radar systems hold several advantages such as reduced blind-angle [2], high range resolution, robustness to weather conditions, and lower costs than millimeterwave radars. Nevertheless, if the environment is populated by cars equipped with independent automotive radars, the performance of each radar will be degraded by interference signals entering their receivers, which are originated from nearby radars operating at the same frequency. The performance degradation can be mitigated with the use of cross-polarization and code division multiple access (CDMA) technology [3-5], however, quantitative evaluation of interference suppression have not been fully exploited. The authors previously studied the interference rejection capability of automotive radars modulated by maximum length sequence (MLS) codes [6], and derived the basic capability of the method to suppress interference.

In this paper, we further investigated the interference suppression performances of UWB automotive radars modulated by pseudo random sequence using binary MLS, and Gold sequences (GS). Both sequences were selected due to their good auto- and cross-correlation properties, which is significantly useful in suppressing interference from nearby radars. Initially, the desired-to-undesired signal power ratio $(D U R)$ was examined when two cars pass by each other on the opposite side of the road. Suppression of interference was numerically evaluated by calculating the probability of false alarm $\left(P_{f a}\right)$, and the probability of detection $\left(P_{d}\right)$ of the radar for the estimated DUR. Important characteristics of both sequence which is related to the automotive radar system design was also discussed, such as the order of shift register, code length, number of available sequence, and auto- and cross-correlation properties.

\section{Calculation of Desired-to-Undesired Signal Power Ratio}

We estimated the DUR while assuming a scenario where two cars pass each other on a road. Both cars are equipped with UWB radars aiming horizontally and $60^{\circ}$ from the axial direction of travel of the cars, as depicted in Fig. 1. The desired signal power $D$ is the receiving radar signal emitted from its own transmitter and reflected from the oncoming car; and the undesired signal power $U$ is the interference signal entering the receiver from the other radar equipped on the oncoming car. Here, $D$ and $U$ are 


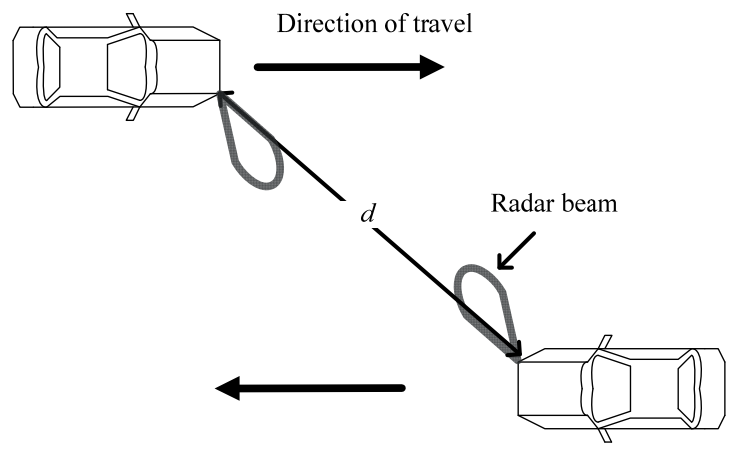

Fig. 1. Scenario for evaluating a range of $D U R$.

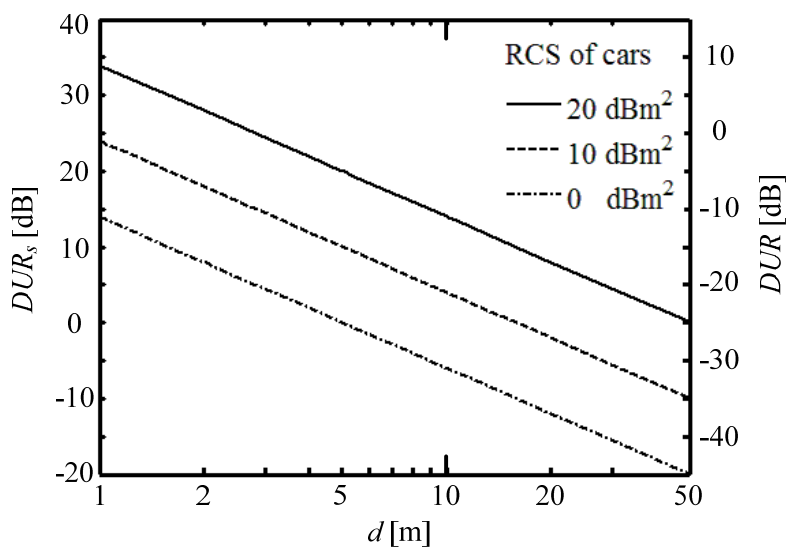

Fig. 2. Substantial $D U R_{s}$ against the distance between two cars, assuming $45^{\circ}$-polarization for transmission and reception.

expressed by:

$$
D=\frac{P_{t} G_{t}(\theta) G_{r}(\theta) \lambda_{0}^{2} \sigma}{(4 \pi)^{3} d^{4}}[\mathrm{~W}]
$$

and

$$
U=P_{t} G_{t}(\theta) G_{r}(\theta)\left(\frac{\lambda_{0}}{4 \pi d}\right)^{2}[\mathrm{~W}]
$$

where $P_{t}$ is the transmitting power, $G_{t}$ the antenna gain for transmission and reception, $G_{r}$ the azimuth angle from the antenna boresight, $\lambda$ the wavelength and $\sigma$ the radar cross section (RCS) of targets, and $d$ the distance between the two cars. Therefore, the DUR is expressed by

$$
D U R=10 \log \left(\frac{D}{U}\right)=10 \log \left(\frac{\sigma}{4 \pi d^{2}}\right)[\mathrm{dB}] .
$$

Here, we could understand from (3) that the DUR is independent of $P_{t}, G_{t}(\theta), G_{r}(\theta)$, and when the two radars transmit the same power and use the same antennas. Equations (1) to (3) were derived based on free space (FS) model consideration. This is based on calculations of first Fresnel zone radius (refer Appendix) and assumptions that the directivity of antenna beam was optimized for vehicular applications. Furthermore, UWB two-ray propagation model reported in [7] indicated that frequency diversity effects reduced the fading depth from two-ray interference for the case of UWB, approximating the case of FS propagation. Hence, we considered the FS model in this study.
The RCS of a typical passenger car ranges from 2 to $20 \mathrm{dBm}^{2}$ [2], and $d$ varies from 1 to $20 \mathrm{~m}$, considering the lane widths of roads range from 2.8 to $3.5 \mathrm{~m}$. It is important to note that we used a simplified assumption of UWB RCS based on investigation in [2]. Precise estimation of UWB RCS for cars may require considerations of antenna height and wavelength, however, the authors limit the scope of the paper to evaluating the radar performance, and will address the mentioned problem in a separate work.

If the two radars utilize vertical and horizontal polarization, the DUR lies between -35 and $9 \mathrm{~dB}$. Since the $D U R$ of $-35 \mathrm{~dB}$ (when $\sigma=2 \mathrm{dBm}^{2}$ and $d=20 \mathrm{~m}$ ) is too low to discriminate between the desired and undesired signals, we further assume that the radars use $45^{\circ}$-inclined linear polarization. In this case, the desired signal is received in $45^{\circ}$ polarization, while the undesired signal in $-45^{\circ}$ polarization. Here, assuming the cross polarization discrimination of the antennas to be $X P D \mathrm{~dB}$, the substantial $D U R\left(D U R_{s}\right)$ is therefore enhanced by

$$
D U R_{s}=D U R+X P D .
$$

Here, we consider the typical XPD of linear polarized antennas such as slot and microstrip patches exceeds $25 \mathrm{~dB}$. Therefore, the $D U R_{s}$ ranges approximately from -10 to $34 \mathrm{~dB}$. The plot of $D U R_{s}$ against distance $d$ is shown in Fig. 2.

\section{Simulation Setup}

\subsection{Pulse Trains Modulated with Maximum Length- or Gold-Sequences}

The UWB radar under consideration transmits a series of UWB pulses which is modulated by pseudo random sequence consisting of binary phase shift keying (BPSK) codes with either MLS or GS. The pulse waveform can be arbitrarily selected to comply with UWB spectrum mask specified by regulatory bodies. When the radar is using pulses with duty cycle $\tau_{d}=133 \mathrm{~ns}$, for example, the detection range $R=c \tau_{d} / 2=20 \mathrm{~m}$, where $c$ is the electromagnetic wave velocity. The time interval for alarming the driver was set to be $100 \mathrm{~ms}$, considering normal human reaction time [8]. During this $100 \mathrm{~ms}$, the radar is capable to transmit 750,000 pulses $(=100 \mathrm{~ms} / 133 \mathrm{~ns})$.

For the case of MLS, the codes are generated with a $k$-stage feedback shift register, resulting in $2^{k}-1$ code length. Therefore, the pulse train will be sufficiently repeated within the $100 \mathrm{~ms}$, as shown in Fig. 3. In this study, $k$ was chosen to vary from $7,9,11$ to 13 , in order to evaluate the impact of code length on the radar performance. Allocation of different MLSs to different radars facilitates the suppression of interference from other radars, if the respective MLSs have good cross-correlation properties. To achieve this condition, MLS feedback taps generating preferred pairs of MLS were selected and assigned to each 


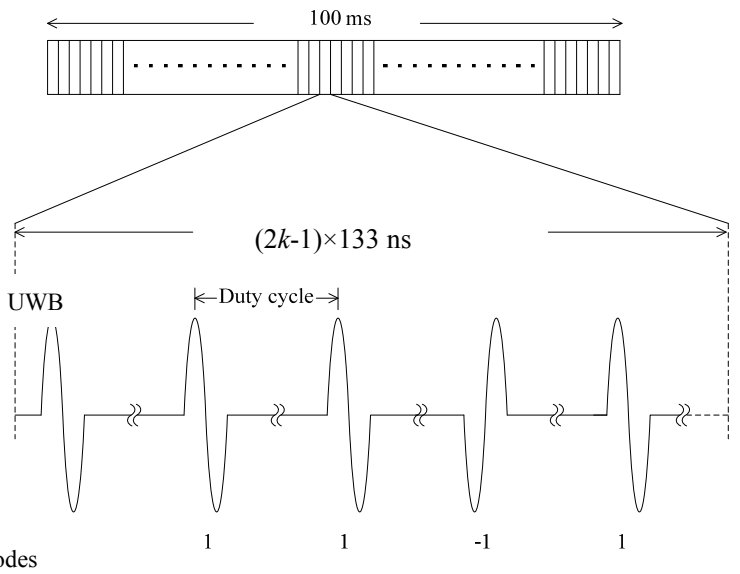

Psuedo codes

representation

Fig. 3. Example of a BPSK-modulated pulse train with an MLS.

\begin{tabular}{|c|c|c|}
\hline Order of MLS code, $\boldsymbol{k}$ & $\begin{array}{c}\text { MLS feedback } \\
\text { taps (MLS1) }\end{array}$ & $\begin{array}{c}\text { MLS feedback taps } \\
\text { (MLS2) }\end{array}$ \\
\hline 7 & $(3,7)$ & $(1,2,3,7)$ \\
\hline 9 & $(4,9)$ & $(3,4,6,9)$ \\
\hline 11 & $(2,5,8,11)$ & $(2,3,10,11)$ \\
\hline 13 & $(1,3,4,13)$ & $(4,5,7,9,10,13)$ \\
\hline
\end{tabular}

Tab. 1. Selected MLS feedback taps to generate preferred pairs of MLS

each radar, as listed in Tab. 1. The total number $N$ of the preferred pairs of MLSs, however, is limited: e.g. $N=6,2$, 4 , and 4 when $k=7,9,11$, and 13, respectively. An MLS yields a two-level autocorrelation function:

$$
\theta(\tau)= \begin{cases}2^{k}-1 & \text { if } \tau=0 \\ -1 & \text { if } \tau \neq 0\end{cases}
$$

and a preferred pair of MLSs possesses a relatively low level cross correlation function. On the other hand, GSs possess no particular preferred pairs. GSs possess $(2 k-1)$ different sequences. GSs provide relatively low three-level cross correlation function $\left(2^{(k+1) / 2}-1,-1\right.$, and $\left.-2^{(k+1) / 2}-1\right)$. The autocorrelation function of the GSs of a length of $k$ is given by

$$
\theta(\tau)=\left\{\begin{array}{cr}
2^{k}-1 & \text { if } \tau=0 \\
\text { either } 2^{(k+1) / 2}-1,-1, \text { or }-2^{(k+1) / 2}-1 & \text { if } \tau \neq 0
\end{array}\right.
$$

The larger fluctuating $\theta(\tau)$ (when $\tau \neq 0$ ) is a drawback of the GSs. Accordingly, auto correlation and cross correlation of GS is slightly inferior to it of MLS. However, GS possess far more different sequences. Therefore, utilization of GS can suppress interference from multiple other radars. On the other hand, in either case of random sequence, the selection of $k$ and feedback taps should be negotiated among the cars beforehand, for instance, via an inter-vehicle communication link. The design of physical layer and communication protocol for this purpose is not studied within this paper, since it is outside the scope of the present study.

\subsection{Simulation Structure}

A series of numerical simulation was carried out to evaluate the probability of false alarm $\left(P_{f a}\right)$ and probability of detection $\left(P_{d}\right)$ of the UWB radar system. Signal flow diagrams and representative waveforms are presented in Fig. 4. Figure 4(a) depicts a case that the receiver receives the desired signal alone, generated from a unique code designated by either MLS1 or GS1. Additive white Gaussian noise (AWGN) was injected into the signal at the receiver input port. Signal-to-noise power ratio (SNR) was fixed at $10 \mathrm{~dB}$. For the sake of presentation clarity, the matched filter output was normalized by the maximum value of auto-correlation function $(2 k-1$ for a $k$-stage MLS and GS), as shown in Fig. 4(a). Figure 4(b) depicted the case where the undesired signal $U$ alone entered the receiver, in which $P_{f a}$ was calculated by varying the $D U R$ and threshold level. Figure 4(c) represents the signal flow and the waveforms when both the desired and undesired signals co-exist within the same channel. The $P_{d}$ was calculated under the presence of undesired signal $U$ in the system, when $P_{f a}$ was fixed $10^{-2}$ or $10^{-4}$ for $D U R$ between -10 to $30 \mathrm{~dB}$, and the length of sequences $L=127,511$, 2047, 8191 (corresponding to $k=7,9,11$, and 13, respectively). The maximum antenna gain, obtained from product of transmission and reception was assumed to be $11 \mathrm{dBi}$, and the half-power beamwidth is $60^{\circ}$ (common value for typical slot antennas).

The simulation was carried out based on the following conditions:

- Matched filter output was normalized by the maximum value of autocorrelation function in Fig. 4(a).

- The same normalization was applied to the matched filter output in Figs. 4(b) and (c).

- The $P_{f a}$ was calculated by varying $D U R$ and the threshold level in Fig. 4(b).

- The $P_{d}$ was calculated when $P_{f a}$ was fixed at $10^{-2}$ or $10^{-4}$.

\section{Simulation Results}

First, the false alarm probability $P_{f a}$ was calculated when no target is present in the system, and the receiver receives only the undesired signal $U$ generated by MLS2 or GS2 from the interfering radar. The matched filter output in Fig. 4(a) was normalized for a certain level of the desired signal $D$ by the maximum value $(2 k-1)$ of auto-correlation function of MLS1 and GS1. Similarly, the matched filter output in Fig. 4(b) was normalized in a similar manner, where the $U$ was varied at a ratio between -30 to $10 \mathrm{~dB}(-10 \leq D U R[\mathrm{~dB}] \leq 30)$ to the desired signal level $D$. The calculated $P_{f a}$ for $L=127,511,2047,8191$ (corresponding to $k=7,9,11$, and 13 , respectively), are plotted 


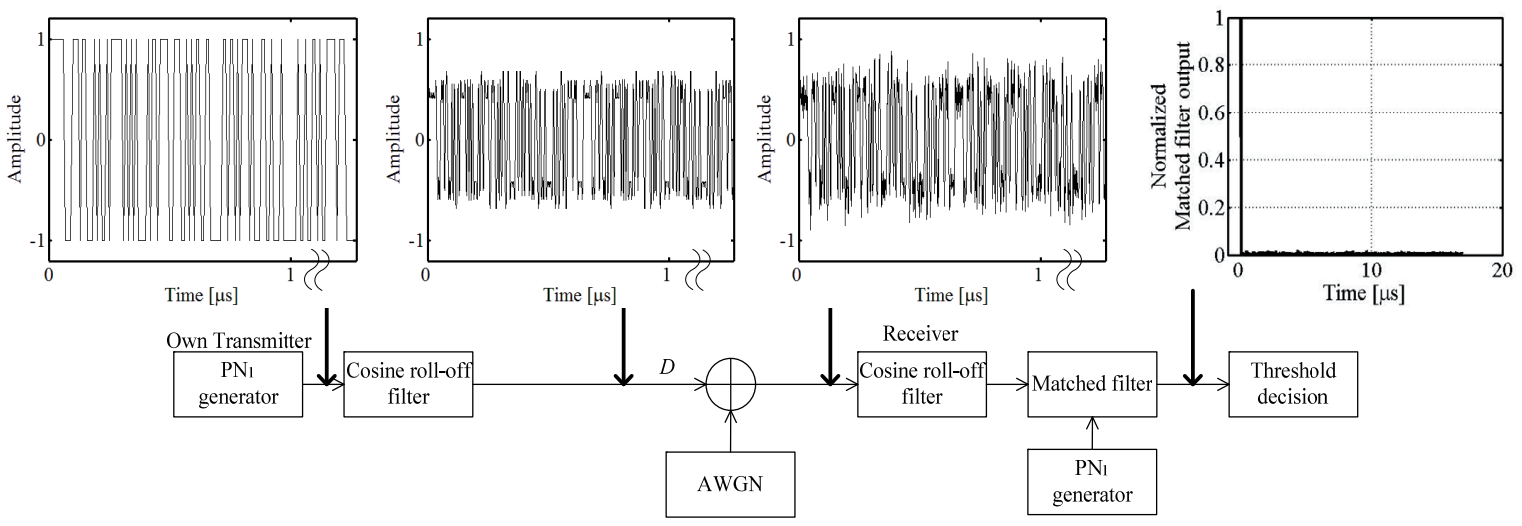

(a)
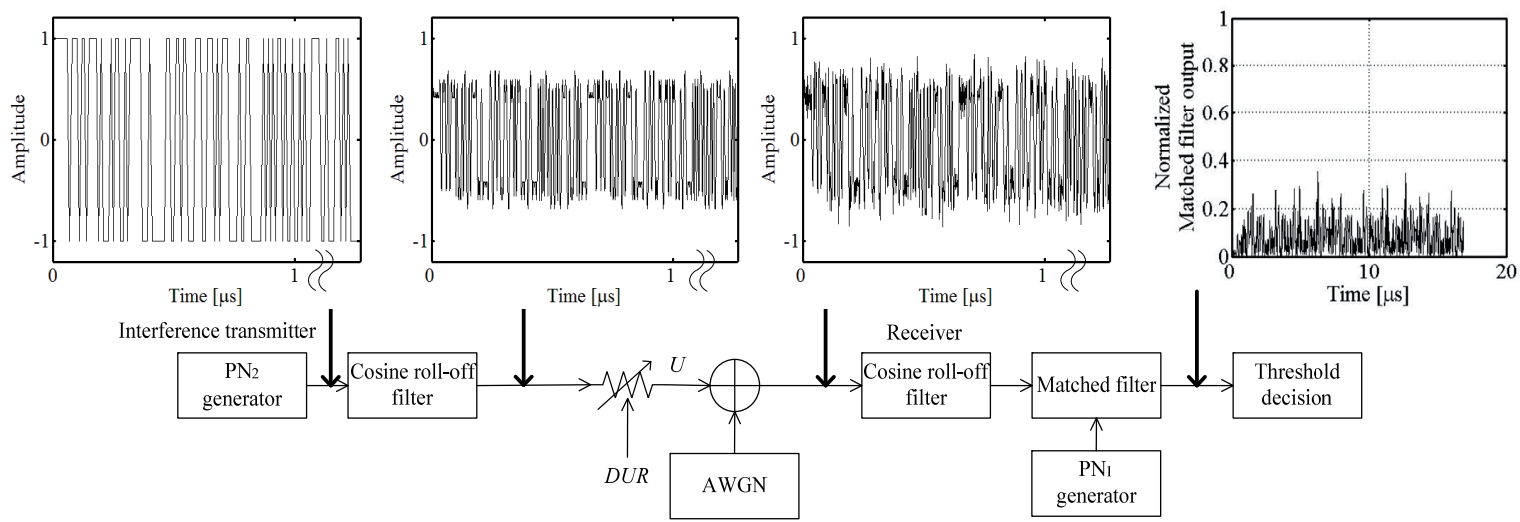

(b)

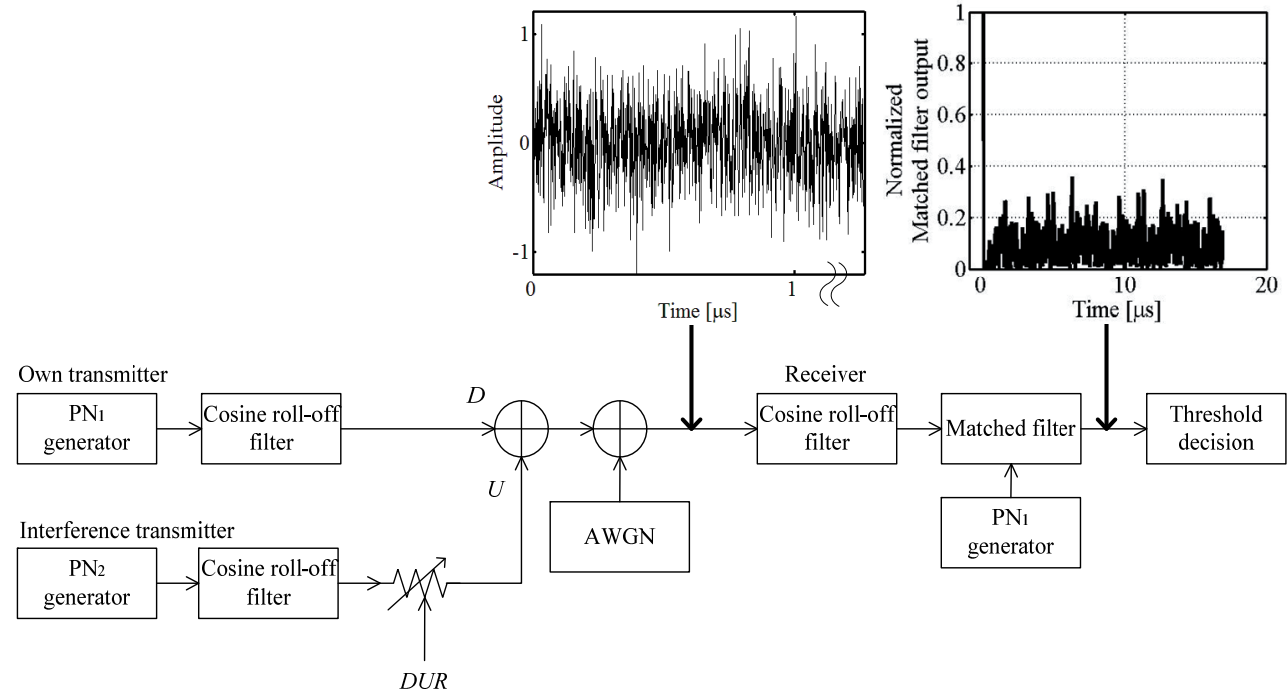

(c)

Fig. 4. Block diagrams and simulated waveforms used in the study. The receiver receives: (a) the desired signal from host vehicle radar transmitter, (b) the undesired signal from the other radar transmitter, or (c) the desired and the undesired signals simultaneously.

in Figs. 5 and 6 between $-10 \leq D U R \leq 10 \mathrm{~dB}$. It can be observed from the figure that higher $D U R$ contributes to decrease in $P_{f a}$ yielding the same threshold level. It was also found that larger $k$ results in the lower threshold level yielding the same $P_{f a}$. The $P_{f a}$ was almost the same when comparing MLS and GS. It is considered that an effect of low three-level cross correlation was lost because AWGN was super-imposed to the signal at the receiver input.
Probability of detection for both cases of MLS and GS were calculated by using the threshold level when both desired and undesired signals existed at $-10 \leq D U R \leq$ $30 \mathrm{~dB}$. Figures 7 and 8 show the simulation results of $P_{d}$ when $P_{f a}=10^{-2}$ and $10^{-4}$. The MLS and GS modulation resulted in significant improvement of $P_{d}$ in comparison with no use of MLS and GS. For the case of MLS, improvements relative to no use of MLS were approximately 


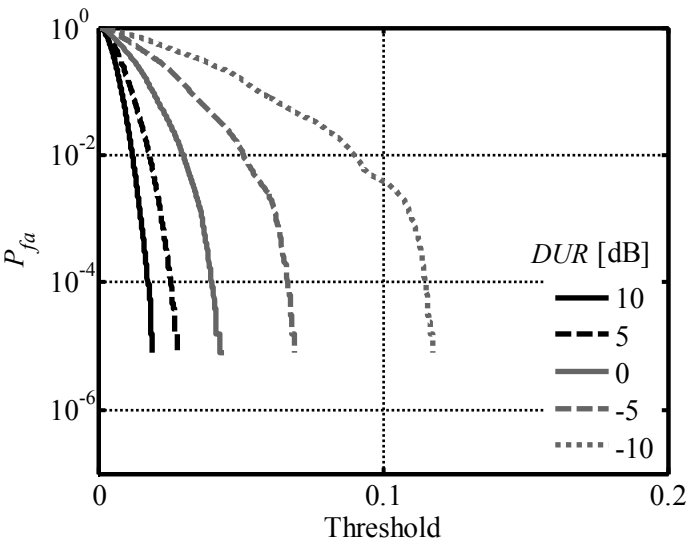

(a)

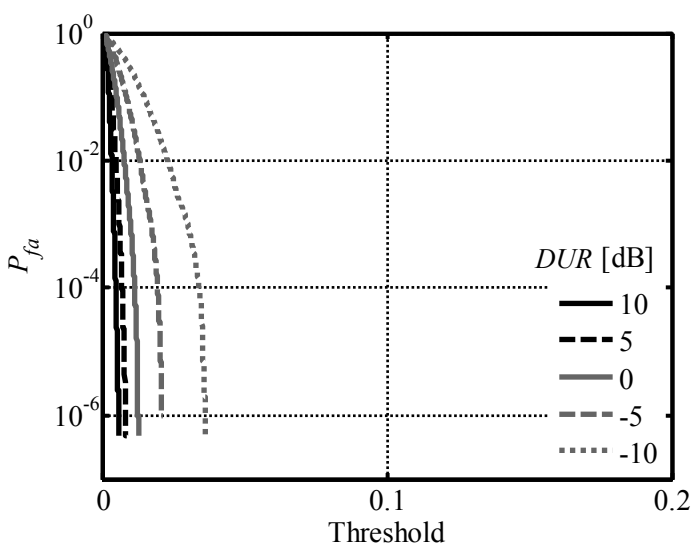

(c)

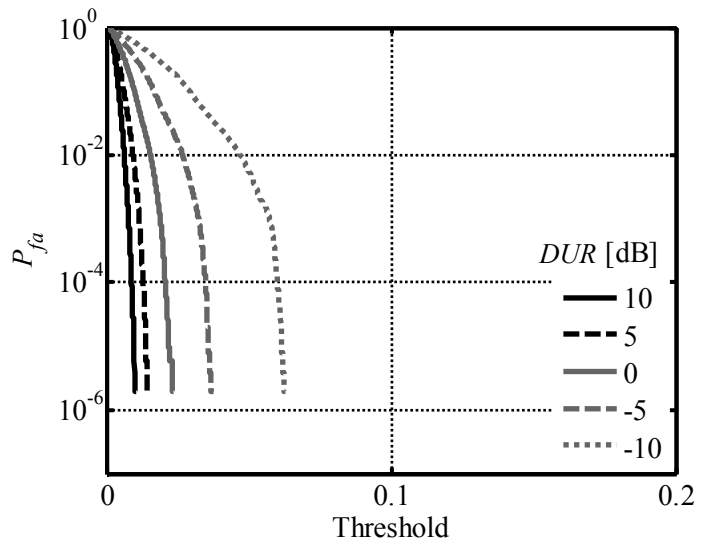

(b)

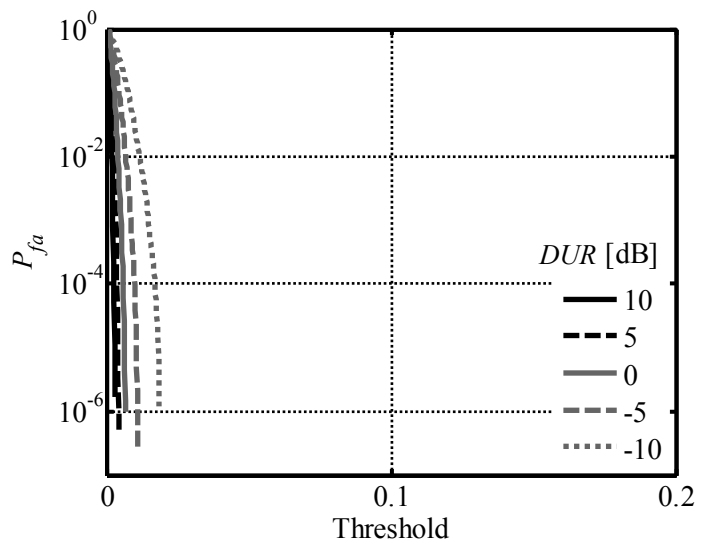

(d)

Fig. 5. Simulated false alarm probability: length of MLS = (a) 127, (b) 511, (c) 2047, and (d) 8191 .

$21,27,33$, and $39 \mathrm{~dB}$ for $L=127,511,2047,8191$, respectively. This is because the maximum values of autocorrelation function of MLS are $127(21 \mathrm{~dB}), 511(27 \mathrm{~dB})$, $2047(33 \mathrm{~dB})$, and $8191(39 \mathrm{~dB})$ for $L=127,511,2047$, 8191, respectively. Addition of the order of MLS stages $k$ by 2 induced approximately $5 \mathrm{~dB}$ reduction of $D U R$ yielding the same level of $P_{d}$.

Improvements relative to no use of GS were approximately 23, 27, 33, and $40 \mathrm{~dB}$ for $L=127,511,2047$, 8191, respectively, as plotted in Fig. 8. Similar to the case of MLS, improvement of approximately $5 \mathrm{~dB}$ of $P_{d}$ was observed by increasing the length of the GS codes. Figure 9 compares both the $P_{d}$ of MLS and GS. The detection

\begin{tabular}{|c|c|c|c|}
\hline$D U R[\mathrm{~dB}]$ & $\boldsymbol{P}_{f a}$ & $M L S$ & $G S$ \\
\hline 30 & $\begin{array}{l}10^{-2} \\
10^{-4}\end{array}$ & $\begin{array}{l}0.999 \\
0.999\end{array}$ & $\begin{array}{l}0.999 \\
0.999\end{array}$ \\
\hline 10 & $\begin{array}{l}10^{-2} \\
10^{-4}\end{array}$ & $\begin{array}{l}0.995 \\
0.993\end{array}$ & $\begin{array}{l}0.995 \\
0.993\end{array}$ \\
\hline-10 & $\begin{array}{l}10^{-2} \\
10^{-4}\end{array}$ & $\begin{array}{l}0.963 \\
0.946\end{array}$ & $\begin{array}{l}0.963 \\
0.945\end{array}$ \\
\hline
\end{tabular}

Tab. 2. Typical detection probability: length of MLS and GS $=8191$. probabilities were found almost the same for MLS and GS, the former of which yielded slightly higher performances, as typically summarized in Tab. 2. The performance improvement marked by usage of GS was larger when the $D U R$ was smaller (for example $D U R=-10 \mathrm{~dB}$ ). This fact is in parallel with the previous studies of comparisons between MLS and GS in bit error rate and peak-to-noise ratio in the fields of spread spectrum communication [9] and radar [10], respectively.

\section{Conclusion}

This paper presented a quantitative evaluation of the performance of radars employing pseudo random sequences -MLS and GS, for suppressing interference from nearby radars. The study was carried out through numerical simulations that were constructed based on a link budget considering a scenario where two cars equipped with independently operating UWB radar pass each other on a road. Assuming important parameters for the scenario such as $45^{\circ}$-inclined polarization and distance $d$ between the two passing cars, $D U R$ was found to vary approximately from -10 to $30 \mathrm{~dB}$. The UWB radar under consideration transmits a UWB pulse train that is modulated by BPSK with 
either MLS or GS to suppress interference from the radar of the other passing car. Allocation of different MLSs and GSs to different radars facilitates the suppression of interference from other radars. Threshold level was determined to yield false alarm rates of either at $10^{-2}$ or $10^{-4}$ (caused by interference from the other radar) when no return signal was received from its own radar transmitter. The employment of MLS or GS to modulate the radar pulse train resulted in significant improvement in detection probability. Improvements relative to no use of MLS were approxi-

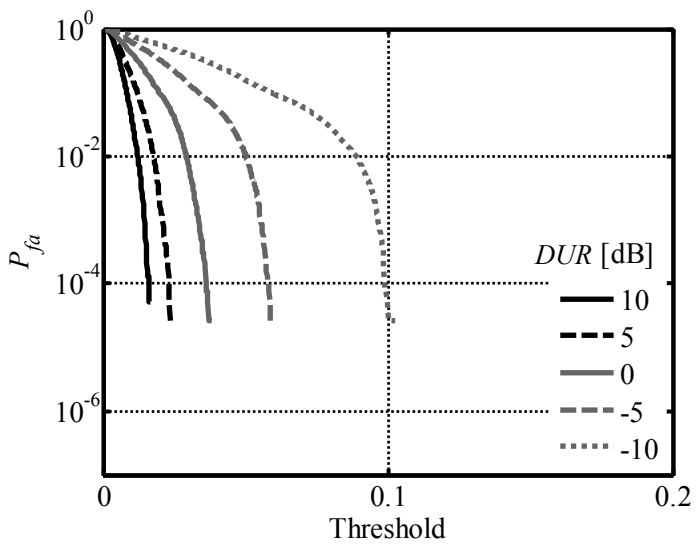

(a)

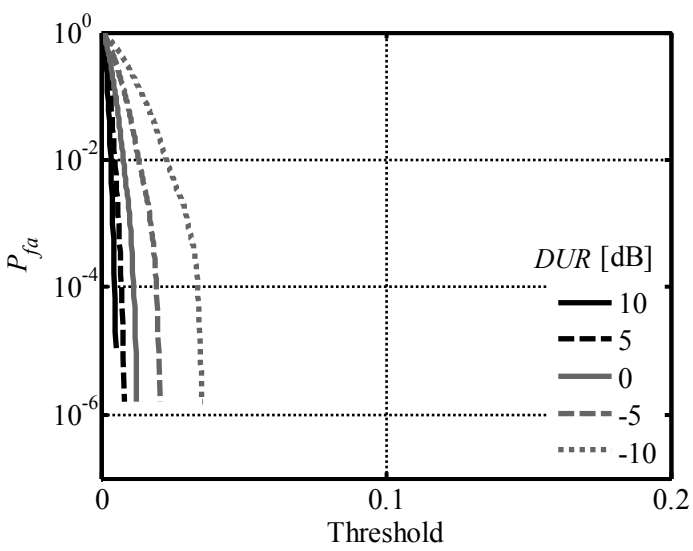

(c) mately $21,27,33$, and $39 \mathrm{~dB}$ for $L=127,511,2047,8191$, respectively. Improvements relative to no use of GS were approximately $23,27,33$, and $40 \mathrm{~dB}$ for $L=127,511$, 2047,8191 , respectively. The detection probabilities were found almost the same between MLS and GS. These results show that GS is comparable to MLS when applied to the spread-spectrum vehicular radars, however it is more advantageous considering the larger number of available sequences. Both MLS and GS marked improvements of $P_{d}$ with increasing code length.

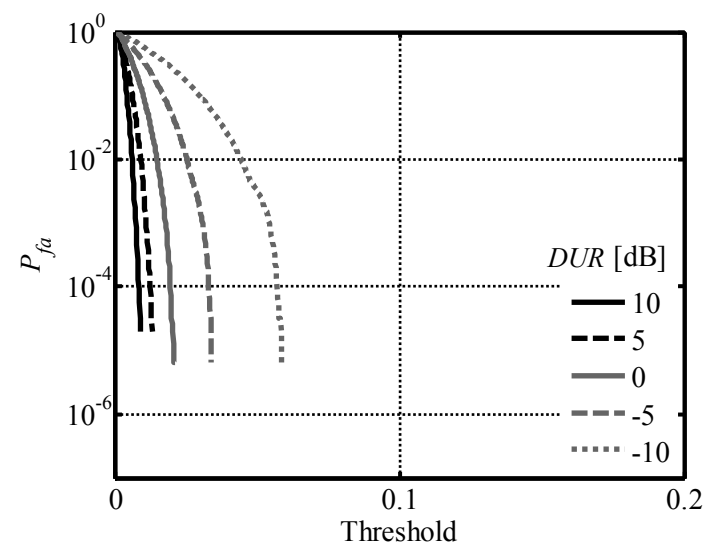

(b)

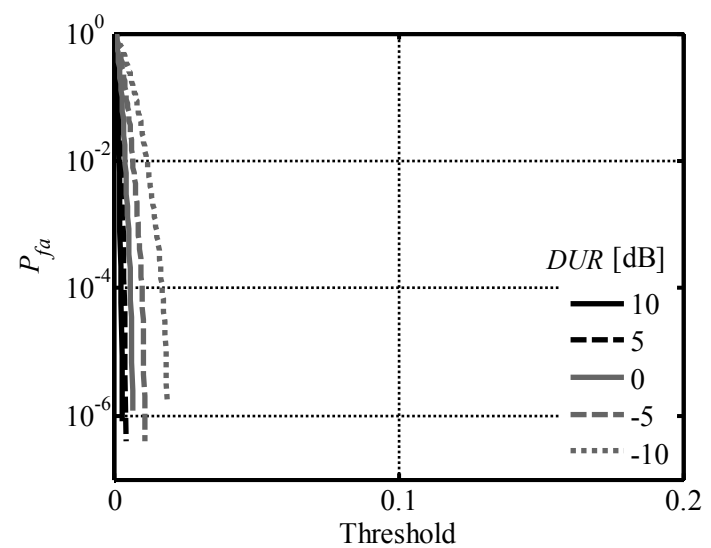

(d)

Fig. 6. Simulated false alarm probability: length of $\mathrm{GS}=$ (a) 127 , (b) 511 , (c) 2047 , and (d) 8191 .

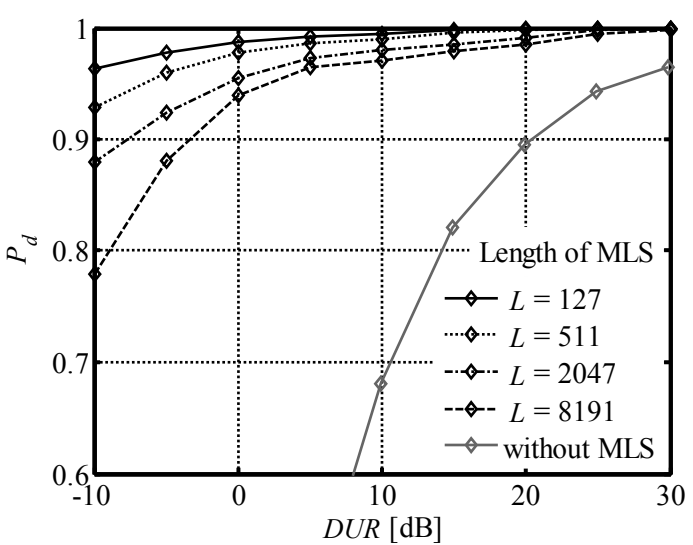

(a)

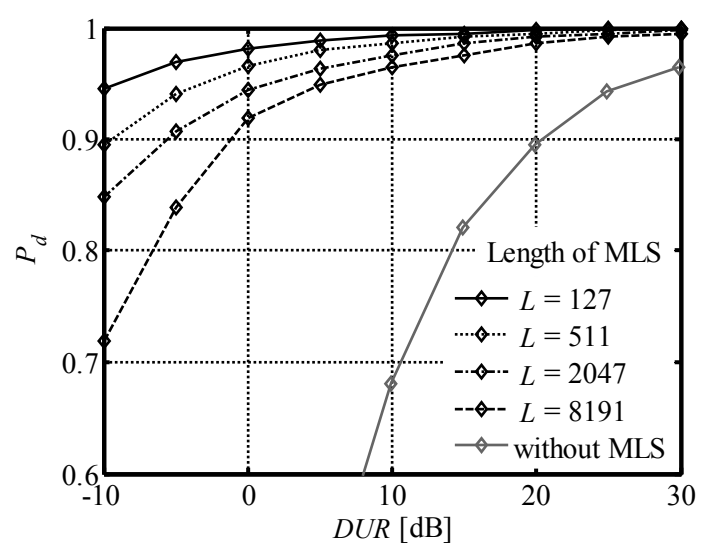

(b)

Fig. 7. Simulated detection probability of MLS: when $P_{f a}=$ (a) $10^{-2}$ and (b) $10^{-4}$. 


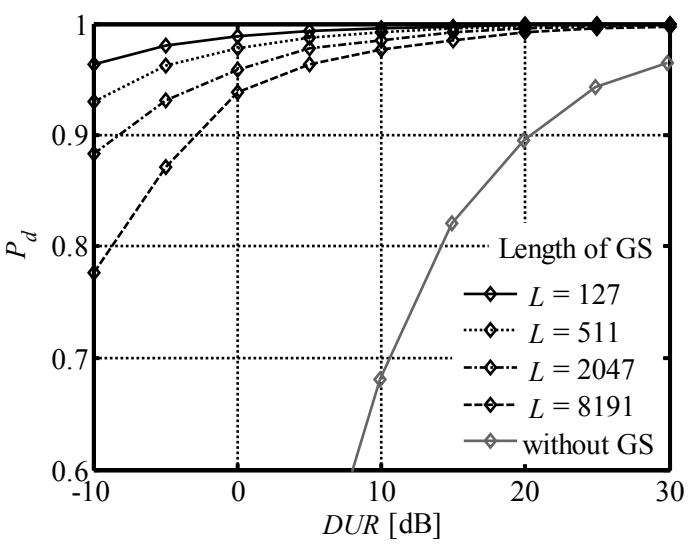

(a)

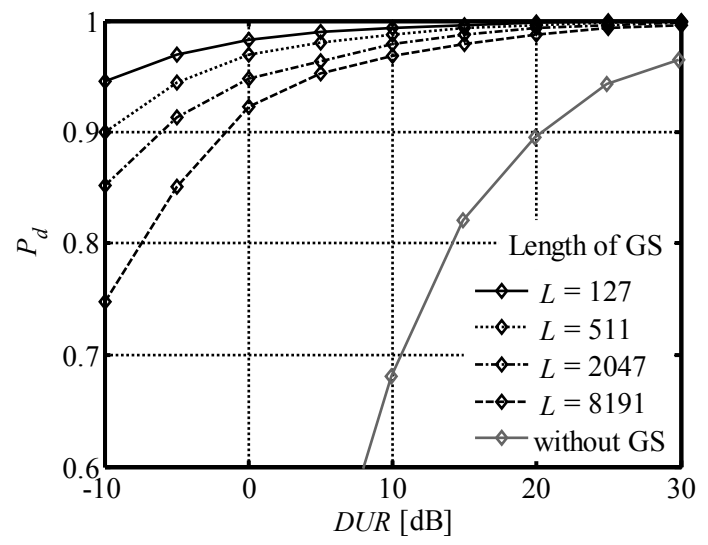

(b)

Fig. 8. Simulated detection probability of GS: when $P_{f a}=$ (a) $10^{-2}$ and (b) $10^{-4}$.

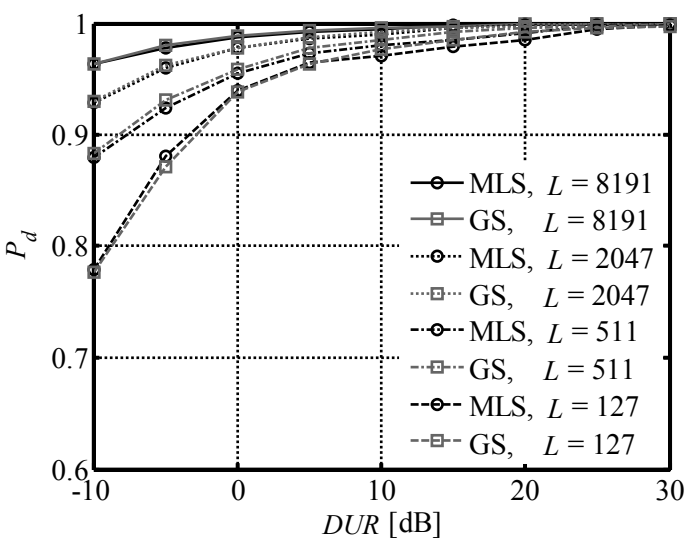

(a)

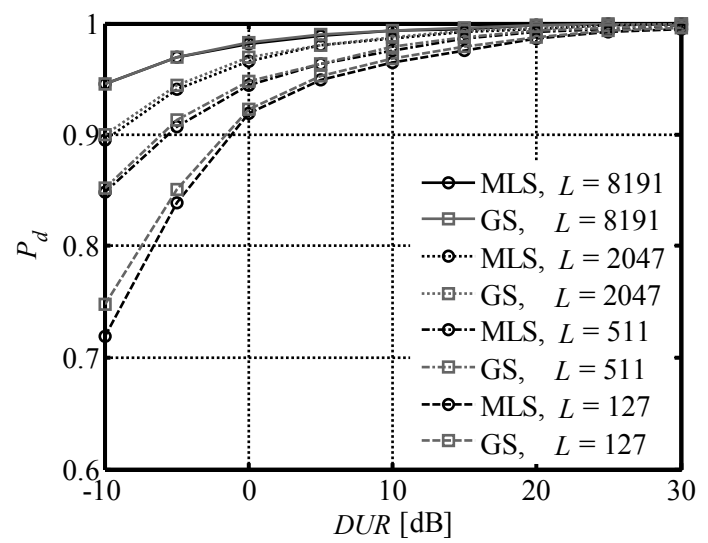

(b)

Fig. 9. Comparison of $P_{d}$ between MLS and GS: when $P_{f a}=$ (a) $10^{-2}$ and (b) $10^{-4}$.

\section{Appendix}

The first Fresnel zone radius when operating the UWB radar at $25 \mathrm{GHz}$ of center frequency. The $n^{\text {th }}$-Fresnel zone radius can be calculated using the following equation,

$$
F_{n}=\sqrt{\frac{n \lambda d_{1} d_{2}}{d_{1}+d_{2}}}
$$

where $d_{1}$ and $d_{2}$ is the distance from the transmitting and receiving antennas to the center of the Fresnel zone, $\lambda$ is the wavelength, and $n$ is the index number for the $n^{\text {th }}$-Fresnel zone radius. Assuming the distance $10 \mathrm{~m}, d_{1}$ and $d_{2}$ are both $5 \mathrm{~m}$. Centre frequency of $25 \mathrm{GHz}$ gives us $\lambda=12 \mathrm{~mm}$, hence, $F_{1}=0.17 \mathrm{~m}$. The transmitting and receiving antennas heights of $0.5 \mathrm{~m}$ are sufficient for the system to exclude a destructive reflected wave from the ground that could reduce the receiving power.

\section{References}

[1] GRESHAM, I., JENKINS, A., EGRI, R., et al. Ultra-wideband radar sensor for short-range vehicular applications. IEEE
Transactions on Microwave Theory and Techniques, 2004, vol. 52, no. 9, p. 2105-2120. DOI: 10.1109/TMTT.2004.834185

[2] KOBAYASHI, T., TAKAHASHI, N., YOSHIKAWA, M., et al. Measurement of automobile UWB radar cross sections at Ka band. In Ultra-Wideband, Short-Pulse Electromagnetics 7, New York (United States), Springer, 2007, p. 586-592. ISBN: 978-0-38737728-5

[3] GAMBI, E., SPINSANTE, S. Chaos-based radars for automotive applications: theoretical issues and numerical simulation. IEEE Transactions on Vehicular Technology, Nov. 2008, vol. 57, no. 6, p. 3858-3863. DOI: 10.1109/TVT.2008.921632

[4] WEI, Y., LIU, L., ZHANG, J. Blind estimation of PN sequence of DS-CDMA signal in multipath. In Proceedings of the 2nd International Conference on Communications and Networks. Yichang (China), 2012, p. 1695-1699. DOI: 10.1109/CECNet.2012. 6201645

[5] FISH, A., GUREVICH, S. Performance estimates of the pseudorandom method for radar detection. In Proceedings of the 2014 IEEE International Symposium of Information Theory. Honolulu (United States), 2014, p. 3102-3106, DOI: 10.1109/ISIT.2014. 6875405

[6] KATO, H., KOBAYASHI, T. Detection probability of automotive radars using maximum length sequences to suppress interference from nearby radars. In Proceedings of the IEEE International Aerospace and Electronics Conference. Dayton (United States), 2014, p. 396-400. DOI: 10.1109/NAECON.2014.7045843

[7] SATO, S., KOBAYASHI, T. Path-loss exponents of ultra 
wideband signals in line-of-sight environments. In Proceedings of the 2004 IEEE Eighth International Symposium on Spread Spectrum Techniques and Applications. Sydney (Australia), 2004, p. 488-492. DOI: 10.1109/ISSSTA.2004.1371748

[8] KARL, I., BERG, G., RUGER, F., et al. Driving behavior and simulator sickness while driving the vehicle in the loop: validation of longitudinal driving behavior. IEEE Intelligent Transportation Systems Magazine, 2013, vol. 5, no. 1, p. 42-57, ISSN: 1939-1390. DOI: 10.1109/MITS.2012.2217995

[9] CHEN, X. H., LANG, T., OKSMAN, J. Multiple chip-rate DS/CDMA system and its spreading code dependent performance analysis. IEE Proceedings- Communication, 1998, vol. 145, no. 5 , p. 371-377. DOI: 10.1049/ip-com:19982289

[10] YANG, C., LI, W., ZENG, Y., et al. Impulse response measurements using Gold sequences. In Proceedings of the 2006 8th International Conference on Signal Processing. Beijing (China), 2006, p. 1-4. DOI: $10.1109 /$ ICOSP.2006.346070

\section{About the Authors ...}

Idnin PASYA became a Member of IEEE in 2010. He received the B.E. and M.E. degrees in Information and Communication Engineering from Tokyo Denki University in 2004 and 2006, respectively, and his Ph.D. degree in Information, Communication and Media Design Engineering from Tokyo Denki University in 2015. He worked as an engineer in Toshiba PC \& Network, Tokyo, Japan, from 2006 to 2009 . He is currently a senior lecturer in the Faculty of Electrical Engineering, Universiti Teknologi MARA, Malaysia. His research interests include evaluation of ultra wideband communication systems, MIMO radar and its applications. Dr. Idnin received the IEEE MTT Best Paper Award in 2014 IEEE Radio and Wireless Symposium.

Hiroshi KATO received his B.E. and M.E. degrees from Tokyo Denki University in 2013 and 2015, respectively. He currently works as an engineer in NEC Communications Systems, Japan. His research interests include UWB automotive radars and signal processing.

Takehiko KOBAYASHI received the B.E., M.E., and Ph.D. degrees in Electrical Engineering from the University of Tokyo in 1978, 1980, and 1983. He joined Nippon Telegraph and Telephone in 1983 and was engaged in research on various wireless communication systems. He was a guest scientist at the National Bureau of Standard (now NIST) in Boulder, Colorado, U. S. A. in 1986. From 1998 to 2001, he was with YRP Key Tech Labs, which focused on the 4 th generation mobile communication systems. Currently, he is a professor at the Department of Information and Communication Engineering, Tokyo Denki University. He received the IEICE Best Paper Awards in 2001 and 2002, the IEICE Achievement Award in 2007, and the Telecom System Awards from the Telecommunications Advancement Foundation in 2003 and 2005. His current research interests include ultra wideband wireless systems, mobile communication channel characterization, and teletraffic evaluation of mobile communication networks. Dr. Kobayashi is a member of IEEE. 